\title{
The Chrispin-Norman score in cystic fibrosis: doing away with the lateral view
}

\author{
C. Benden*, C. Wallis*, C.M. Owens*, D.A. Ridout ${ }^{\mp}$ and R. Dinwiddie*
}

ABSTRACT: The Chrispin-Norman chest radiograph scoring (CNS) system is widely used to assess respiratory disease progression in cystic fibrosis (CF). Frontal and lateral chest radiographs were performed. The present authors developed a modified CNS, which obviates the need for the lateral film. This study compares the original and the current authors' modified scoring system.

A total of 50 chest radiographs from CF children, taken between August and December 2003, were scored according to the original and modified CNS. Two observers scored all 50 chest radiographs, scoring in random order the frontal radiographs, and separately the frontal and lateral radiographs together.

There was no evidence of a difference between the methods for either observer, using the Bland and Altman 95\% limits of agreement as follows: observer 1 (-2.0-1.9), and observer 2 (-1.77-2.2). No evidence of a difference between the observers for either method was found, comparing the 95\% limits of agreement (-5.5-5.7) with the modified CNS (-5.6-6.4).

In conclusion, in terms of the final score, good agreement was found between the use of the original and modified Chrispin-Norman score. In addition, low inter-observer variability was shown for both methods. The use of the modified Chrispin-Norman chest radiograph scoring system to stage disease severity in cystic fibrosis removes the need for a lateral chest radiograph.

KEYWORDS: Chest radiograph scoring system, Chrispin-Norman score, cystic fibrosis

$\mathbf{M}$ onitoring lung structure is an important aspect in the assessment of respiratory disease progression in patients with cystic fibrosis (CF). Conventional chest radiography is currently the most commonly used method of radiological imaging [1, 2] and is recommended annually [3].

Several different scoring systems exist to assess the severity of CF lung disease on chest radiograph and to allow longitudinal follow-up [1,4]. The Chrispin-Norman chest radiograph scoring (CNS) system is widely accepted and demonstrates good reproducibility between observers $[5,6]$. The original CNS involves frontal and lateral chest radiographs, which are inspected for the typical abnormalities seen in CF and scored according to a standardised scoring system [7]. The current authors have developed a modified CNS, which obviates the need for the lateral film and, thus, reduces the radiation burden.

This study compares the original with the modified CNS system to evaluate if the two methods are comparable in terms of the final score.

\section{METHODS}

The first 50 CF children who attended the Great Ormond Street Hospital for Children (London, UK), which is a tertiary CF centre, for annual review around the time of their birthday between August 12003 and December 312003 were included in the study.

Frontal (postero-anterior) and lateral chest radiographs were scored according to the original and modified CNS systems using computerised radiography integrated to a picture archiving and communication system (Siemens, Erlangen, Germany). Two observers (R. Dinwiddie and C. Wallis) scored all 50 chest radiographs. Both observers are consultants in paediatric respiratory medicine, having cared for children with CF in a tertiary CF centre for more than a decade each. Chest radiographs were scored in random order selected by one of the other authors (C. Benden) by chance. The two observers were not involved in the clinical assessment of the CF subjects at the time of their annual review and were, therefore, unaware of the subjects' current clinical status. The chest radiographs of each subject were scored a minimum of 3 days after
AFFILIATIONS

${ }^{*}$ Respiratory Medicine Unit, and

\# Dept of Imaging, Great Ormond

Street Hospital for Children National Health Service Trust, and

- Centre for Paediatric Epidemiology and Biostatistics, Institute of Child

Health, London, UK.

CORRESPONDENCE

C. Benden

Respiratory Medicine Unit

Great Ormond Street Hospital for

Children

London WC1N 3JH

UK

Fax: 442078138440

E-mail: bendec@gosh.nhs.uk

Received:

May 192005

Accepted after revision:

July 282005

SUPPORT STATEMENT

Research at the Institute of Child Health and Great Ormond Street Hospital for Children National Health Service Trust (London, UK) benefits from research and development funding received from the National Health Service Executive. 
the annual review in one session, together with chest radiographs of at least four other subjects as a subgroup (minimum subgroup size $n=5$ ). The two observers initially scored, in separate sessions, the frontal radiograph only, then the frontal and lateral radiographs of each subject together but in random order within the subgroup, not automatically scoring chest radiographs of the same subject, and using both methods in succession. The observers were not aware of the scores of chest radiographs taken at previous annual review clinic visits, as these results were stored in the centre's CF database.

Chest radiographs were evaluated according to the original CNS system, identifying radiological signs of chest overinflation on the lateral radiograph (sternal bowing, diaphragmatic depression, spinal curvature) and parenchymal lung changes on the frontal radiograph (bronchial wall thickening, ring shadows, mottled shadows, large soft shadows). For the latter, the lung fields were divided into the following four zones on the frontal film: right upper, left upper, right lower, and left lower. Scores of 0 (not present), 1 (present but not marked) and 2 (marked) were given according to the severity for each zone in relation to each of the individual parenchymal lung changes. Further details regarding the radiological signs of parenchymal lung changes in CF are described elsewhere [7]. For the modified CNS system, hyperinflation is assessed by the degree of overinflation seen on the frontal radiograph alone, using the shape of the rib cage, darkness of lung parenchyma indicating air trapping, and level of the diaphragm (table 1).

\begin{tabular}{|c|c|c|c|c|}
\hline \multicolumn{2}{|l|}{ Feature } & Not present & $\begin{array}{c}\text { Present but not } \\
\text { marked }\end{array}$ & Marked \\
\hline \multicolumn{5}{|c|}{ Overinflation } \\
\hline Diaphr & ic depression & 0 & 1 & 2 \\
\hline Chest & nape & 0 & 1 & 2 \\
\hline Lung fi & & 0 & 1 & 2 \\
\hline \multicolumn{5}{|c|}{ Bronchial line shadows } \\
\hline Right 4 & zone & 0 & 1 & 2 \\
\hline Left up & & 0 & 1 & 2 \\
\hline Right Ic & zone & 0 & 1 & 2 \\
\hline Left lov & & 0 & 1 & 2 \\
\hline \multicolumn{5}{|c|}{ Ring shadows } \\
\hline Right u & zone & 0 & 1 & 2 \\
\hline Left up & & 0 & 1 & 2 \\
\hline Right Ic & zone & 0 & 1 & 2 \\
\hline Left lov & & 0 & 1 & 2 \\
\hline \multicolumn{5}{|c|}{ Mottled shadows } \\
\hline Right $u$ & zone & 0 & 1 & 2 \\
\hline Left up & & 0 & 1 & 2 \\
\hline Right Ic & zone & 0 & 1 & 2 \\
\hline Left lov & & 0 & 1 & 2 \\
\hline \multicolumn{5}{|c|}{ Large soft shadows } \\
\hline Right $u$ & zone & 0 & 1 & 2 \\
\hline Left up & & 0 & 1 & 2 \\
\hline Right Ic & zone & 0 & 1 & 2 \\
\hline Left lov & & 0 & 1 & 2 \\
\hline
\end{tabular}

At the current authors' institution, which is dedicated to lowdose paediatric techniques, the effective radiation doses range from $0.003 \mathrm{mSv}$ for 1-yr-old subjects to $0.01 \mathrm{mSv}$ in $15-\mathrm{yr}$-old subjects for a frontal chest radiograph, and $0.008 \mathrm{mSv}$ for $1-\mathrm{yr}-$ old subjects and $0.015 \mathrm{mSv}$ in 15-yr-old subjects for a lateral chest radiograph. Effective dosages were calculated using National Radiological Protection Board (NRPB) computer software (NRPB-SR279; NRPB, Chilton, UK) for specific paediatric and adolescent phantoms.

Bland and Altman limits of agreement were calculated to demonstrate the agreement between the two methods for each observer in turn, and a paired t-test was used to compare the two methods. Similarly, limits of agreement were calculated for each method to assess agreement between observers [8] An intra-class correlation coefficient (ICC) was calculated to indicate the agreement between observers for each method. ICC is expressed on a $0-1$ scale, where 0 represents no agreement and 1 represents perfect agreement [9]. The assumptions of normality were checked using the ShapiroFrancia test [10].

This prospective study was approved by the Great Ormond Street Hospital for Children National Health Service Trust and Institute of Child Health Research Ethics Committee (London, $\mathrm{UK})$.

\section{RESULTS}

\section{Study population}

The study sample included 30 female and 20 male children with CF. The median age at the time of recruitment was $8.9 \mathrm{yrs}$ (range 0.6-15.6 yrs). Thirty-five patients were $\Delta \mathrm{F} 508$ homozygous, and the remaining 15 had other genotypes. In total, 17 patients were chronically infected with Pseudomonas aeruginosa. Patient characteristics are given in table 2.

\section{Chest radiograph scoring results}

There was no evidence of a difference between the two methods for either observer. The mean difference $(95 \%$ limits of agreement) between methods for observer 1 was $-0.06(-2.0$ 1.9) $(p=0.67)$. For observer 2 , the mean difference $(95 \%$ limits of agreement) was $0.24(-1.7-2.2)(\mathrm{p}=0.09$; fig. 1$)$.

No evidence of a difference between the observers was found for either method, although the limits of agreement were slightly narrower for the original CNS compared with the modified CNS. The mean difference (95\% limits of agreement)

\section{TABLE 2 Patient characteristics}

Characteristics

$\begin{array}{lc}\text { Subjects } \mathbf{n} & 50 \\ \text { Males/females } \mathbf{n} & 20 / 30 \\ \text { Age yrs } & 8.9(0.6-15.6) \\ \text { FEV } \mathbf{1} \% \text { pred } & 80(29-147) \\ \text { FVC \% pred } & 80(32-148) \\ \text { Body mass index } & 16.5(13.1-22.3)\end{array}$

Data are presented as $\mathrm{n}$ and median (range). FEV1: forced expiratory volume in one second; \% pred: \% predicted; FVC: forced vital capacity. 

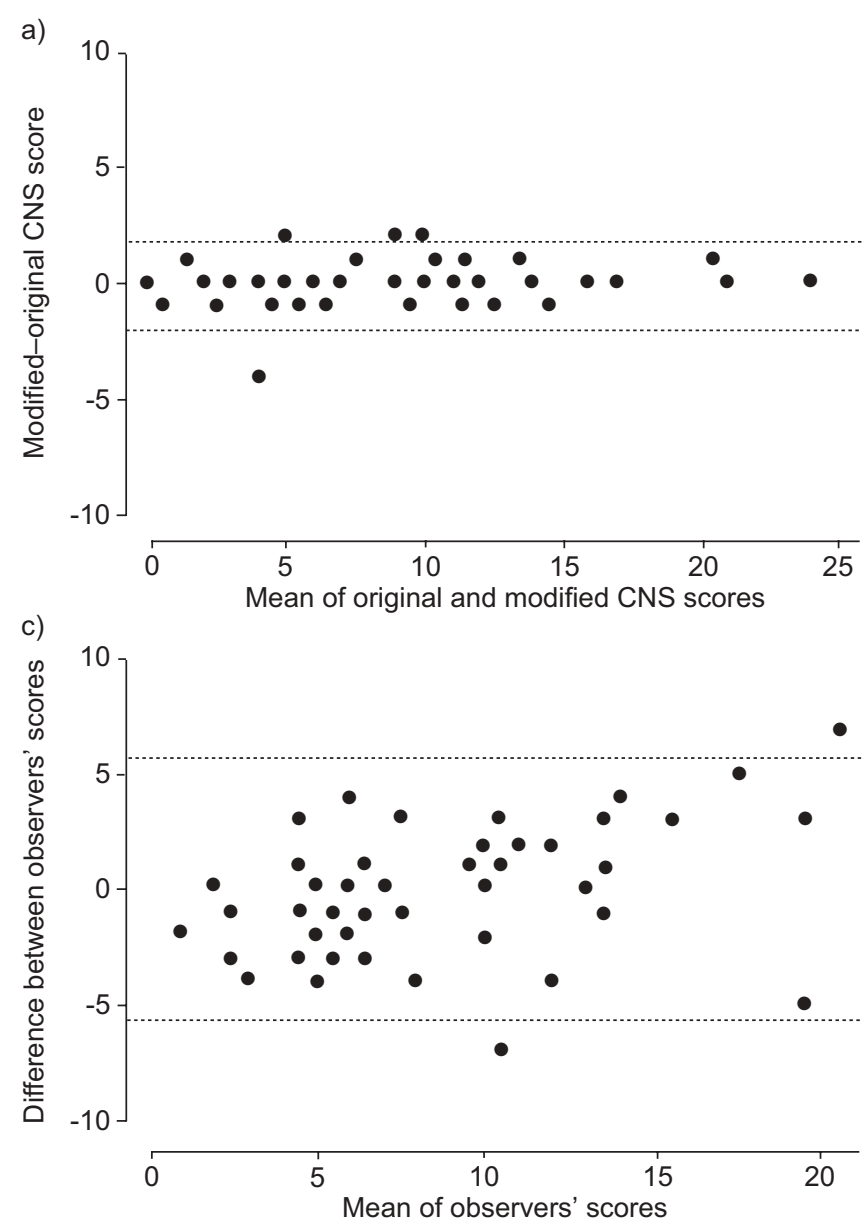

b)

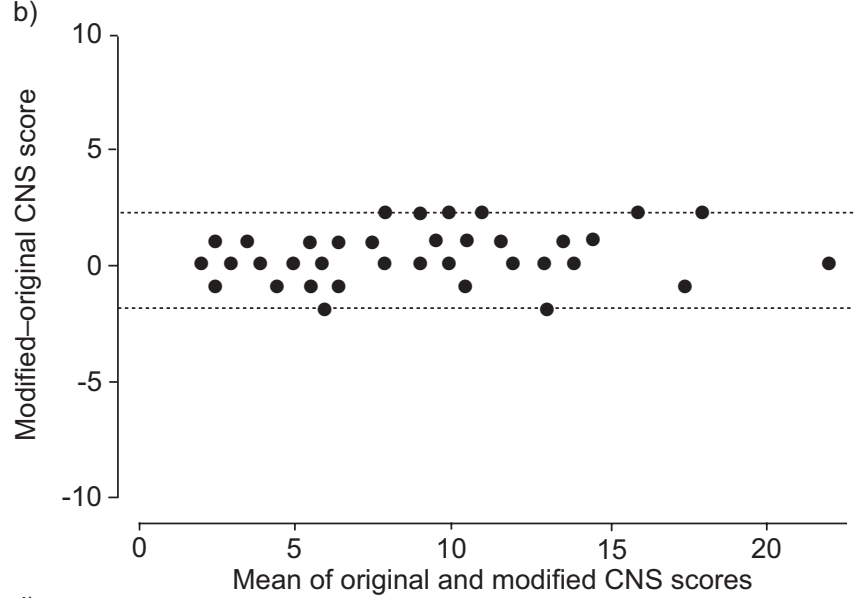

d)

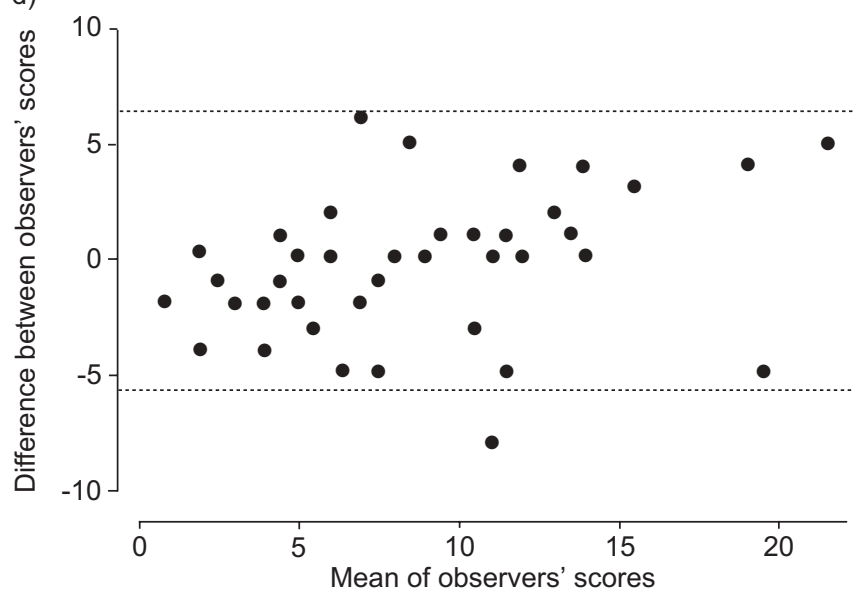

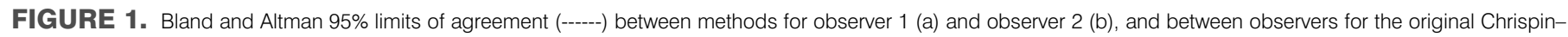
Norman scores (CNS; c) and the modified CNS (d).

between observers for the original CNS was $0.12(-5.5-5.7)$ $(\mathrm{p}=0.76)$. For the modified CNS, the mean difference $(95 \%$ limits of agreement) was $0.42(-5.6-6.4)(p=0.32$; fig. 1$)$.

In addition, there was good agreement between the two observers for both methods using an intra-class correlation coefficient (original CNS 0.92, modified CNS 0.91).

Furthermore, there was more variability between the two observers than there was between the two different methods.

The use of only the frontal chest radiograph for scoring was less time consuming, but the exact time required for each single scoring was not recorded.

A case of a 12-yr-old female CF patient with advanced lung disease is illustrated with frontal and lateral chest radiographs in figure 2.

\section{DISCUSSION}

The European CF Consensus Committee recommended recently that children and adults with $\mathrm{CF}$ should have an annual chest radiograph and the use of chest radiograph scoring systems is advisable. Scoring systems that only require a frontal film were to be used [3]. Furthermore, survival has steadily improved in CF over the last few decades, with a median survival age $>33$ yrs, and is likely to improve further in the future [11]. Since the probability of inducing malignancy by radiation exposure is highest in the paediatric age group [12], and chest radiography involves radiation exposure of the breast tissue of females [13] and increases with each successive chest radiograph, the radiation dose at each exposure has to be kept to an absolute minimum. The "as low as reasonably achievable" ("ALARA") principle warrants citation in reference to radiation-exposure levels. ALARA is not simply a phrase, but a work principle and culture of professional excellence. It is assumed that any exposure to ionising radiation carries some risk. This risk is estimated to be linear. Therefore, it is mandatory to stay as far below the exposure limits to ionising radiation as possible [14]. Radiation-exposure levels of chest radiographs depend on the number of views taken. The frontal (postero-anterior) view causes $\sim 25 \%$ and the lateral view $\sim 75 \%$ of the total effective dose equivalent of the chest radiograph in two views, which ranges $0.06-0.25 \mathrm{mSv}$, depending on the voltage and film-screen system used or the signal to noise ratio in digital systems [13].

This is the first study to report a comparison of the original and modified CNS system. Good agreement between the use of both scoring systems was found. A low inter-observer variability was also shown. 

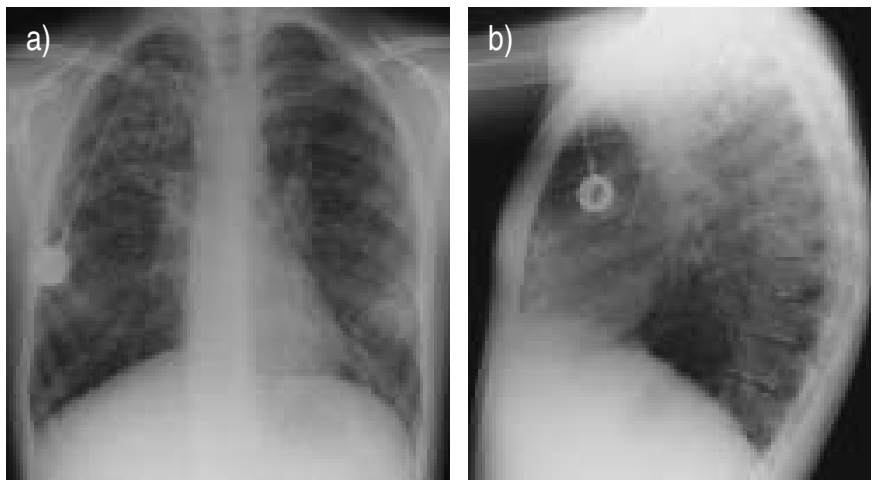

FIGURE 2. a) Frontal and b) lateral computerised chest radiographs of a 12-yrold female cystic fibrosis patient with advanced lung disease and chronic Pseudomonas aeruginosa lung infection. The patient's lung function was as follows: forced expiratory volume in one second $1.35 \mathrm{~L}$ (47\% predicted), and forced vital capacity $2.35 \mathrm{~L}(71 \%$ predicted). There is a Port-A-Cath device in situ. The original Chrispin-Norman chest radiograph score was 21 , and the modified score 21

It is important to note that all scoring systems are subjective and have limitations. A valid scoring system must have significant inter- and intra-observer reproducibility and minimise subjective interpretations [2]. The CNS system is widely used and a recent correlation of six different CF chest radiograph scoring systems with clinical parameters showed low inter-observer variability and good correlation with lung function parameters, especially for the CNS [5].

The use of the modified Chrispin-Norman chest radiograph scoring system removes the need for a lateral chest radiograph to stage disease severity in cystic fibrosis and limits patients' radiation burden. The modified Chrispin-Norman chest radiograph scoring system compares accurately with its predecessor for the purposes of longitudinal assessment.

\section{ACKNOWLEDGEMENTS}

The authors acknowledge the invaluable contributions of $\mathrm{R}$. Evans and C. Pao for the initial pilot investigations that led to the present study.

\section{REFERENCES}

1 Dinwiddie R. Cystic fibrosis. In: Dinwiddie $\mathrm{R}$, ed. Diagnosis and Management of Paediatric Respiratory
Disease. Edinburgh, Churchill Livingstone, 1997; pp. 213214.

2 Conway SP, Littlewood JM. Cystic fibrosis clinical scoring system. In: Dodge JA, Brock DJH, Widdicombe JH, eds. Cystic Fibrosis - Current Topics. John Wiley \& Sons Ltd, 1996; pp. 330-358.

3 Kerem E, Conway S, Elborn S, Heijerman H. Standards of care for patients with cystic fibrosis: a European consensus. J Cyst Fibros 2005; 4: 7-26.

4 Conway SP, Pond MN, Bowler I, et al. The chest radiograph in cystic fibrosis: a new scoring system compared with the Chrispin-Norman and Brasfield scores. Thorax 1994; 49: 860-862.

5 Terheggen-Lagro S, Truijens N, van Poppel N, Gulmans V, van der Laag J, van der Ent C. Correlation of six different cystic fibrosis chest radiograph scoring systems with clinical parameters. Pediatr Pulmonol 2003; 35: 441-445.

6 Matthew DJ, Warner JO, Chrispin AR, Norman AP. The relationship between chest radiographic scores and respiratory function tests in children with cystic fibrosis. Pediatr Radiol 1977; 5: 198-200.

7 Chrispin AR, Norman AP. The systematic evaluation of the chest radiograph in cystic fibrosis. Pediatr Radiol 1974; 2: 101-105.

8 Bland JM, Altman DG. Statistical methods for assessing agreement between two methods of clinical measurement. Lancet 1986; 8: 307-310.

9 Shrout PE, Fleiss JL. Intraclass correlations: uses in assessing rater reliability. Psychol Bull 1979; 86: 420-428.

10 Royston P. A pocket-calculator algorithm for the ShapiroFrancia test for non-normality: an application to medicine. Stat Med 1993; 12: 181-184.

11 Cystic Fibrosis Foundation Patient Registry. 2003 annual data report. Bethesda, Cystic Fibrosis Foundation, 2004.

12 Brenner DJ, Elliston CD, Hall EJ, Berdon WE. Estimated risks of radiation induced fatal cancer from pediatric $\mathrm{CT}$. AJR Am J Roengenol 2001; 176: 289-296.

13 Diederich S, Lenzen $H$. Radiation exposure associated with imaging of the chest: comparison of different radiographic and computed tomography techniques. Cancer 2000; 89: Suppl. 11, 2457-2460.

14 Willis CE, Slovis TL. The ALARA concept in pediatric CR and DR: dose reduction in pediatric radiographic exams - a white paper conference. AJR Am J Roentgenol 2005; 184: 373-374. 